\title{
Respirador mecánico de emergencia
}

\author{
Facundo Pasquevich ${ }^{1,2}$; Alejandro Patanella ${ }^{1}$, Guillermo Garaventta ${ }^{1}$, Marcos Actis ${ }^{1}$ \\ ${ }^{1}$ Centro Tecnológico Aeroespacial - Grupo de Ensayos Mecánicos Aplicados, Facultad de \\ Ingeniería - UNLP; 2 facundo.pasquevich@ing.unlp.edu.ar
}

Resumen. El escenario observado en diferentes países indica que la posible saturación del sistema de salud y la consecuente falta de respiradores para pacientes graves con patología COVID-19, representa un aumento directo en el grado de mortalidad de la enfermedad. El respirador mecánico de emergencia busca establecer un medio de ventilación mecánica que permite proveer presión positiva de ventilación pulmonar a pacientes que no pueden respirar o que tienen problemas para respirar por su propia cuenta. Este sistema sirve para asistir a pacientes en condiciones de emergencia médica grave. Logrando un soporte temporario, mientras se consigue estabilizar al paciente y derivarlo a un respirador mecánico avanzado que permite controlar más variables médicas y el adecuado tratamiento terapéutico.

El objetivo general es el diseño de un respirador mecánico de presión regulada y volumen controlado (PRVC) a partir de la automatización del uso de un respirador manual o resucitador tipo AMBU. El conjunto permite reemplazar la tarea manual realizada por el personal de salud en la operación de un resucitador $A M B U$ por un sistema autónomo que logre la misma función temporalmente. El uso del AMBU como sistema generador de presión se basa en la necesidad de que los sistemas utilizados cuenten con la aprobación de la ANMAT, por lo que el uso de un AMBU homologado permite acortar los procesos de certificaciones.

Palabras clave: respirador; AMBU; PRVC; COVID-19

Recibido: 30/09/2020 Aceptado: 08/10/2020

DOI: https://doi.org/10.24215/26838559e020 


\section{Emergency ventilator}

Abstract. The scenario observed in different countries indicates against the COVID-19 Pandemic that it could be a possible saturation of the health systems and its consequent lack of respirators for severe patients with COVID-19 pathology, increasing the degree of mortality from the disease. The emergency mechanical ventilator developed wants to establish a means of mechanical ventilation to patients who cannot breathe on their own. The ventilator provides positive pressure of pulmonary ventilation to COVID-19 patients. This system is used to assist patients in serious medical emergency conditions, achieving temporary support, while stabilizing the patient and then transfer him to an advanced mechanical ventilator that allows controlling more medical variables and to give him adequate therapeutic treatment.

The general objective of the development is the design a regulated pressure and volume controlled mechanical respirator $(P R V C)$ based on the automation of a manual respirator or $A M B U$ type resuscitator. This equipment can replace the manual task performed by health personnel in the operation of an $A M B U$ resuscitator by an autonomous system that can temporarily achieve the same function. The use of the $A M B U$, as a pressure generating system, is based in using ANMAT approved equipment, so this will lead in a shorten certification process.

\section{Keywords: ventilator; AMBU, PRVC, COVID-19}

\section{Novedad u originalidad local en el conocimiento}

El principal aspecto innovador de este desarrollo es la automatización del accionamiento de un insumo sumamente común en instalaciones sanitarias como es una bolsa AMBU. Generalmente, el AMBU requiere indefectiblemente que una persona lo accione periódicamente, generando así la presión positiva que insufla los pulmones. Esta acción carece de control, más allá de la propia experiencia del usuario. La automatización planteada en este desarrollo, en conjunción con el sistema de control, permite mejorar y optimizar la utilización de respiradores 
Innovación y Desarrollo Tecnológico y Social (2020) 2 (2): 134-166- Número especial COVID-19

manuales. El hecho de que se puedan establecer las variables de la mecánica respiratoria adecuadas para diferentes pacientes, aumenta la eficacia del AMBU. Asimismo, al contar con un sistema de control retroalimentado, una vez establecidas las características de la mecánica respiratoria necesarias, el sistema se reajusta a las condiciones cambiantes del paciente, respetando siempre las condiciones prefijadas y evitando sobrepresiones o efectos no deseados que puedan dañar el sistema respiratorio. A su vez, al contar con pantallas de visualización, el personal de salud puede observar en tiempo real las lecturas de las variables sensadas, aspecto que es imposible en la utilización que comúnmente se le da al AMBU.

Gracias a que el AMBU toma aire del ambiente, no requiere una instalación de aire comprimido para su funcionamiento. A su vez, permite el agregado de oxígeno, utilizando algún tipo de depósito para el gas y luego conectándolo al respirador. Estos aspectos permiten que el respirador pueda ser utilizado en tiendas de campaña, sin grandes necesidades de infraestructura.

\section{Grado de relevancia}

En una primera instancia, el respirador mecánico de emergencia tiene como objetivo principal atenuar una posible saturación del sistema de salud en lo relacionado con soportes de ventilación artificial para pacientes. 
Si bien este sistema no pretende sustituir completamente a un respirador convencional, este desarrollo busca salvar vidas, dándole una oportunidad adicional a los pacientes graves de COVID-19 que no cuentan con un soporte de ventilación mecánica convencional.

Aunque el respirador mecánico desarrollado posee menores cualidades a los respiradores encontrados comúnmente en salas de terapia intensiva, su utilización permitiría extender la ventilación del paciente en forma básica pero regulada hasta que se logre conectarlo a un respirador mecánico avanzado que permita controlar más variables médicas y logre el adecuado tratamiento terapéutico.

En segunda instancia y como un desarrollo a futuro, este respirador se podría aplicar para el traslado de pacientes desde zonas rurales o alejadas de centros médicos hacia las instalaciones sanitarias correspondientes. Esto se lograría, gracias a que su diseño contempla que el conjunto sea autónomo y se encuentre acotado en una pequeña caja donde se alojan todos sus componentes, salvo el suministro externo de oxígeno. A su vez, gracias a sus baterías, el sistema puede funcionar de forma segura durante 24 horas, por lo que adquiere gran relevancia en su uso en zonas alejadas.

\section{Grado de pertinencia}

Considerando la emergencia sanitaria por la que transita tanto nuestro país como el mundo, existe una diversidad de equipos constituidos por profesionales con 
Innovación y Desarrollo Tecnológico y Social (2020) 2 (2): 134-166- Número especial COVID-19

iniciativas para llevar a cabo el armado de dispositivos de asistencia a pacientes afectados por COVID-19, habida cuenta de una eventual ausencia de respiradores mecánicos convencionales que impida la correcta atención de personas en situación de riesgo.

Tanto en el diseño mecánico como en el electrónico del respirador, se hizo especial hincapié en que los componentes e insumos a utilizar sean, en la medida de lo posible, productos disponibles en el mercado local.

Actualmente, el respirador mecánico de emergencia se encuentra en etapa de ensayo. Esta fase es de suma importancia porque el sistema servirá de soporte de vida a pacientes. Por lo tanto, cada uno de sus componentes y luego el conjunto ensamblado, debe superar diversos ensayos de funcionamiento, durabilidad y confiabilidad.

\section{Grado de demanda}

El desarrollo del respirador mecánico de emergencia fue presentado a la convocatoria realizada por la Agencia Nacional de Promoción de la Investigación, el Desarrollo Tecnológico y la Innovación, a principios de abril de 2020, en concordancia con el inicio de la fase del aislamiento social preventivo y obligatorio en nuestro país. A esta convocatoria se presentaron más de 700 proyectos de diferentes índoles, todos apuntados a dar soluciones a la pandemia COVID-19. Luego de ser evaluados, el respirador logró obtener financiamiento junto con otros 
53 proyectos (https://www.argentina.gob.ar/sites/default/files/covid-19 -ce ip adjudicados.pdf ).

Una vez conocida la adjudicación, diferentes portales y medios de comunicación se hicieron eco de la noticia, poniendo de manifiesto el interés de la sociedad en este desarrollo.

Estos medios realizaron algunas de las siguientes notas:

- https://www.pagina12.com.ar/263135-unidad-coronavirus-los-proyectosseleccionados-de-la-convoca

- https://www.ing.unlp.edu.ar/articulo/2020/5/5/ingenieros de la unlp avanzan en el desarrollo de un novedoso respirador mecanico de emergencia

- https://www.0221.com.ar/nota/2020-5-5-16-47-0-desarrollan-en-la-unlp-unnovedoso-respirador-mecanico-de-emergencia-por-el-covid-19

- https://diariociudadcapital.com/la-unlp-fue-seleccionada-para-desarrollar-unrespirador-mecanico-de-emergencia/

- https://www.youtube.com/watch?v=vljbULI6Nbo

- https://www.infoplatense.com.ar/nota/2020-5-6-10-37-0-ingenieros-de-la-unlpdesarrollan-un-respirador-mecanico-de-emergencia

- https://elcactus.com.ar/2020/05/ingenieros-de-la-unlp-avanzan-en-el-desarrollode-un-novedoso-respirador-mecanico-de-emergencia/

- http://agenhoy.com.ar/en-la-unlp-avanzan-en-el-desarrollo-de-un-novedosorespirador-mecanico/ 
Innovación y Desarrollo Tecnológico y Social (2020) 2 (2): 134-166- Número especial COVID-19

- https://www.telam.com.ar/notas/202005/459957-respirador-mecanico-de-

emergencia-la-plata-coronavirus-salud.html

- https://elteclado.com.ar/nota/8563/tres cientificos de la unlp fueron elegidos para financiar sus investigaciones sobre covid 19/

- https://www.tiemposur.com.ar/nota/tres-cientificos-de-la-unlp-elegidos-parafinanciar-sus-investigaciones-sobre-covid19

- http://www.andigital.com.ar/interes-general/item/85215-la-universidad-publicaavanza-en-el-desarrollo-de-un-novedoso-respirador-mecanico-de-emergencia

- https://www.eldia.com/nota/2020-5-3-6-12-36-platenses-entre-los-cientificos-alos-que-financiaran-estudios-informacion-general

- https://www.grupolaprovincia.com/sociedad/avanzan-con-un-respiradormecanico-de-emergencia-que-descomprimira-tarea-medica-ante-elcoronavirus-489173

Es importante remarcar que al interés mostrado por estos medios de comunicación hay que sumarle el acompañamiento y predisposición por parte de entidades privadas, las cuales se pusieron a disposición del proyecto para poder contribuir a su realización.

\section{Desarrollo del producto}

La primera etapa para el desarrollo del respirador fue establecer los parámetros de la mecánica respiratoria que se deben cumplir para que el mismo sea funcional a 
su objetivo. Para ello, el equipo de trabajo participó de diferentes reuniones con expertos médicos y recopiló diversas bibliografías donde se explicitan los parámetros requeridos específicamente para ventilar a pacientes con COVID-19. Entre estos documentos se encuentran las recomendaciones establecidas por la Sociedad Argentina de Terapia Intensiva (2020) y por la Medicines \& Healthcare Products Regulatory Agency (2020) perteneciente al gobierno del Reino Unido.

Como resultado de esta investigación se estableció el diseño de un respirador mecánico de emergencia de presión regulada y volumen controlado (PRVC) con los siguientes parámetros, alarmas, variables, filtros y sistemas de soporte (Hess y Kacmarek 2014; Cairo 2012; Tobin 2006; Sociedad Argentina de Terapia Intensiva 2020; Medicines \& Healthcare Products Regulatory Agency 2020).

Parámetros de mecánica respiratoria:

- Presión máxima 40 cm H20.

- Presión nominal de funcionamiento $30 \mathrm{~cm} \mathrm{H20.}$

- Tiempo inspiratorio 0,6-0,8 segundos.

- PEEP entre 5-15 cm H20.

- Frecuencia de respiración entre 7-30 por minuto.

- Relación entre Inhalación y Exhalación I/E 1:2.

- Volumen de inhalación (Volumen Tidal): entre $150 \mathrm{~cm} 3$ y $800 \mathrm{~cm} 3$.

- Regulación de Fi02 entre 60\%-100\%. 
Innovación y Desarrollo Tecnológico y Social (2020) 2 (2): 134-166- Número especial COVID-19

Alarmas:

- Desconexión del paciente.

- Presión superior a la establecida.

- Volumen de inspiración menor al establecido.

- Desconexión del equipo de la fuente de energía.

Variables a ser monitoreadas:

- Presión de inspiración.

- Presión de expiración.

- Fracción inspirada de $\mathrm{FiO}_{2}$.

- Control visual del funcionamiento general del equipo.

Sistemas de soporte:

- Batería para funcionamiento por 24 horas.

- Redundancia en alarmas.

Filtros:

- Filtro HMEF antes de la entrada/salida de los tubos de respiración de la boca del paciente.

- Filtros HEPA en la salida del aire expirado.

Asimismo, se definió que el comportamiento del flujo, presión y volumen durante el ciclo de respiración debe responder de la forma que se visualiza en la Figura 1. 
Innovación y Desarrollo Tecnológico y Social (2020) 2 (2): 134-166- Número especial COVID-19
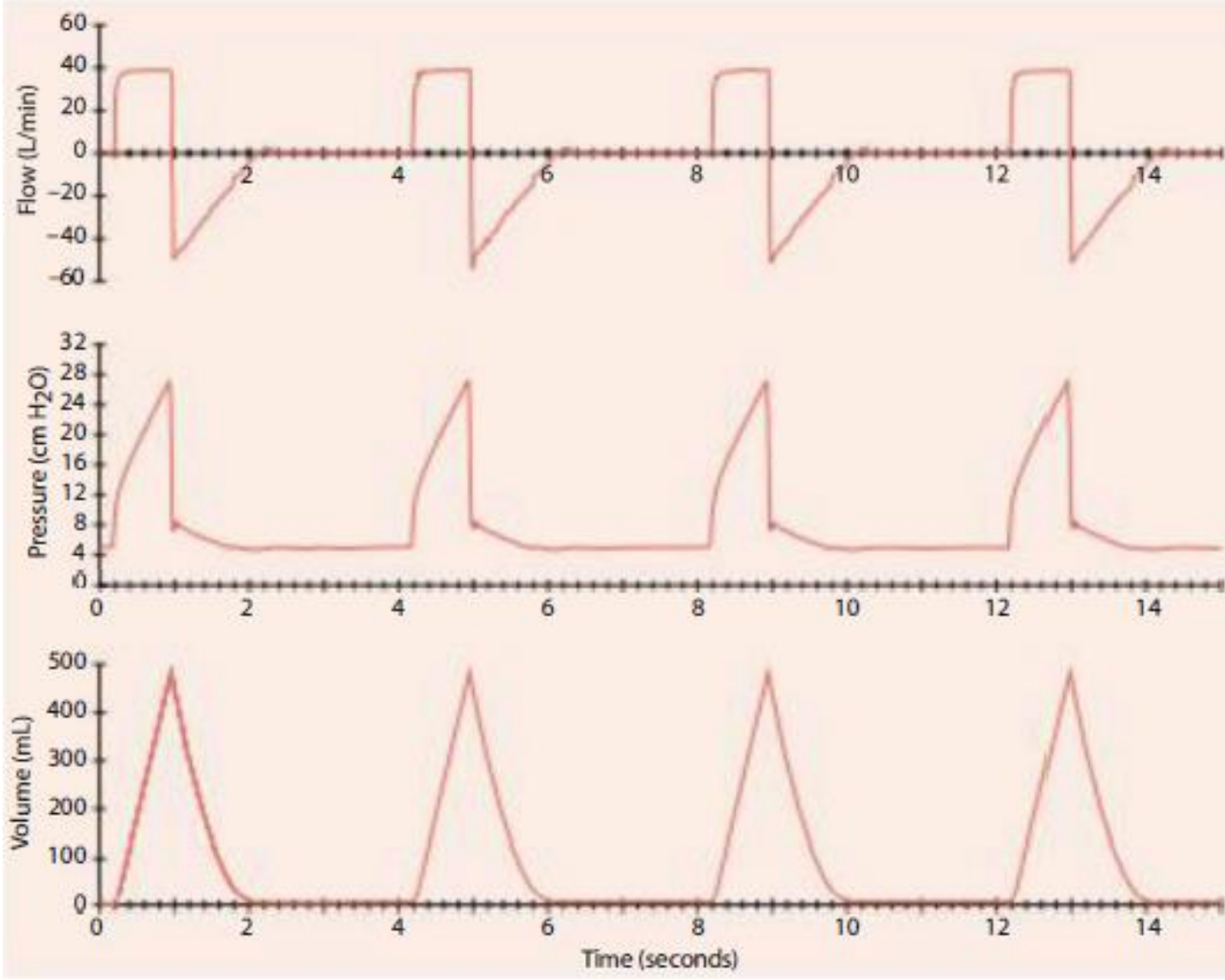

Figura 1. Curvas operacionales típicas. [1,2,3,5,6].

Figure 1. Typical operational curves. $[1,2,3,5,6]$.

Una vez establecidas las características que se especificaron por el grupo de trabajo a la hora del diseño del respirador y considerando la rapidez con que se necesitan obtener resultados debidos a la urgencia que imprime la pandemia, se comenzó una búsqueda de proyectos similares que se estuviesen desarrollando en otras regiones, con el fin de iniciar las tareas con una base de información previamente generada. Asimismo, como parámetro de diseño, se estableció que al ser un equipo de soporte de vida y en base a la necesidad de que sus sistemas cuenten con la 
Innovación y Desarrollo Tecnológico y Social (2020) 2 (2): 134-166- Número especial COVID-19

aprobación de la ANMAT, el respirador debe contar con un AMBU (Artificial Manual Breathing Unit) homologado como fuente generadora de presión ya que su uso permite acortar los tiempos de certificaciones. A modo de ejemplo, en la Figura 2 se observan las bolsas AMBUs utilizadas.

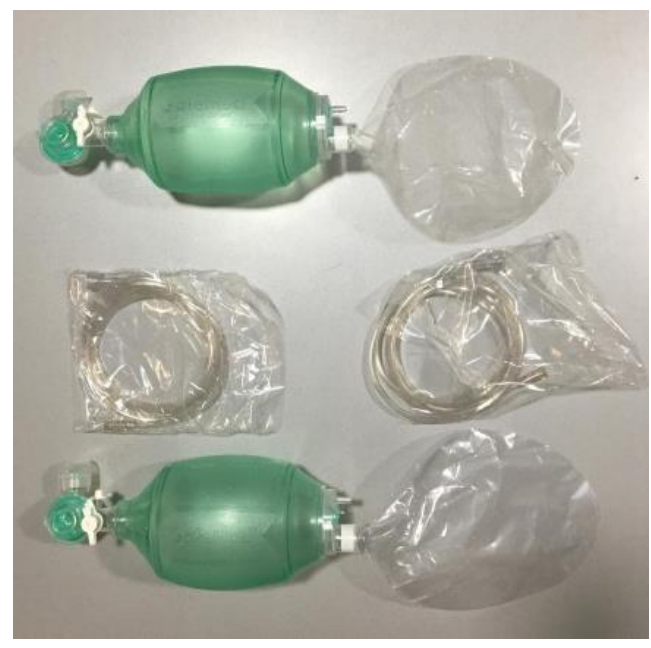

Figura 2. Bolsas AMBUs utilizadas.

Figure 2. AMBUs bags used.

La investigación se centró en equipos preexistentes que tengan la factibilidad de cumplir con las especificaciones de la mecánica ventilatoria establecidas y se concluyó que la mejor opción era tomar como punto de partida al respirador de código abierto que el Massachusetts Institute of Technology estaba desarrollando, bajo el nombre de MIT Emergency Ventilator (MIT 2020).

Tomando como base el MIT Emergency Ventilator (MIT 2020), se prosiguió al diseño conceptual de nuestro respirador, el cual consiste en un mecanismo de engranajes capaz de comprimir una bolsa AMBU logrando que la presión y el volumen de 
Innovación y Desarrollo Tecnológico y Social (2020) 2 (2): 134-166- Número especial COVID-19

inspiración generados en su compresión sigan los parámetros de la mecánica ventilatoria determinados por el operador. Todo el conjunto se encuentra contenido en una caja metálica diseñada para ser transportada de forma manual.

El mecanismo de accionamiento consiste en dos brazos que generan la compresión simétrica del $A M B U$, comandados por un motorreductor que acciona una relación de engranajes. En las Figuras 3 y 4 se presenta un esquema del mecanismo y se indica con las flechas la forma de apriete del AMBU.

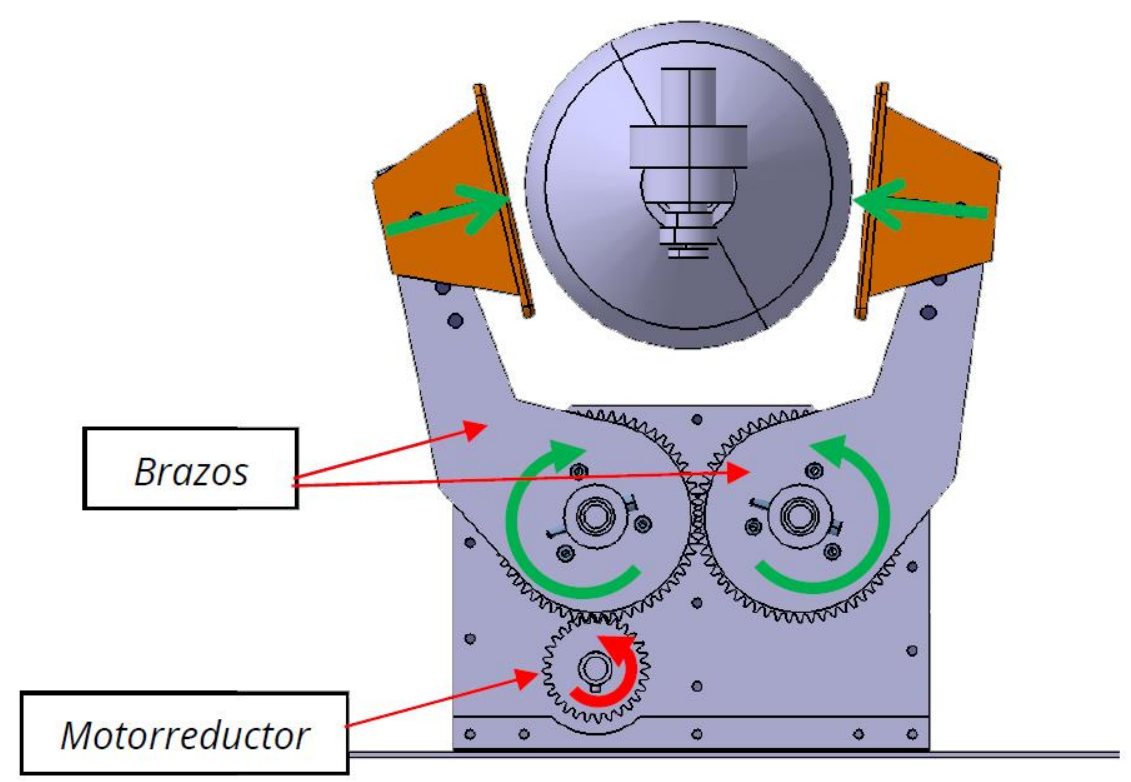

Figura 3. Mecanismo de accionamiento.

Figure 3. Drive mechanism. 


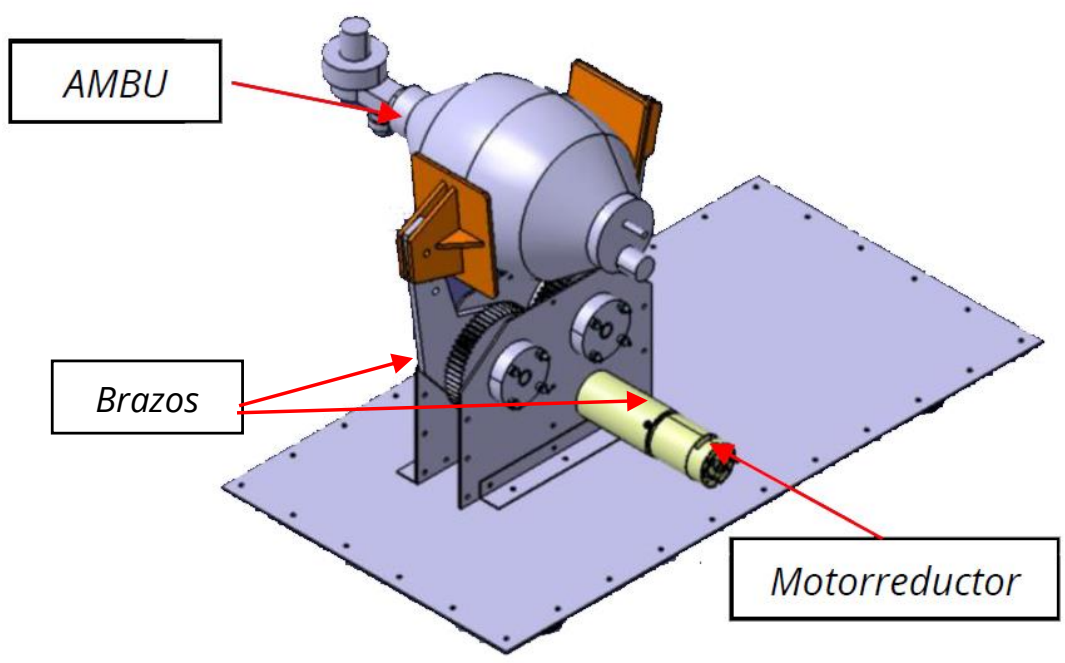

Figura 4. Vista del mecanismo de accionamiento.

Figure 4. Detail of the drive mechanism.

En cuanto al diseño electrónico del sistema de control, este se realiza a partir del uso de microprocesadores y cuenta con pantallas para monitorear las distintas variables de operación y para cambiar parámetros de uso. También, se incorporan diferentes alarmas al sistema para indicar corrimientos en los valores establecidos. Todo el conjunto posee redundancia electrónica para ser tolerante a fallas simples. A su vez, se contempla que su diseño tenga gran flexibilidad para poder ir incorporando alarmas que, según las pruebas y resultados obtenidos, necesiten ser incluidas. Este sistema presenta la posibilidad de adicionar entradas y salidas de medición y control de tal manera de poder conectarlas según cualquier necesidad futura, permitiendo así expandir el desarrollo. 
En la Figura 5 se presenta la arquitectura electrónica básica del MIT Emergency Ventilator (MIT 2020), la cual es análoga en su esencia a la arquitectura de nuestro respirador.

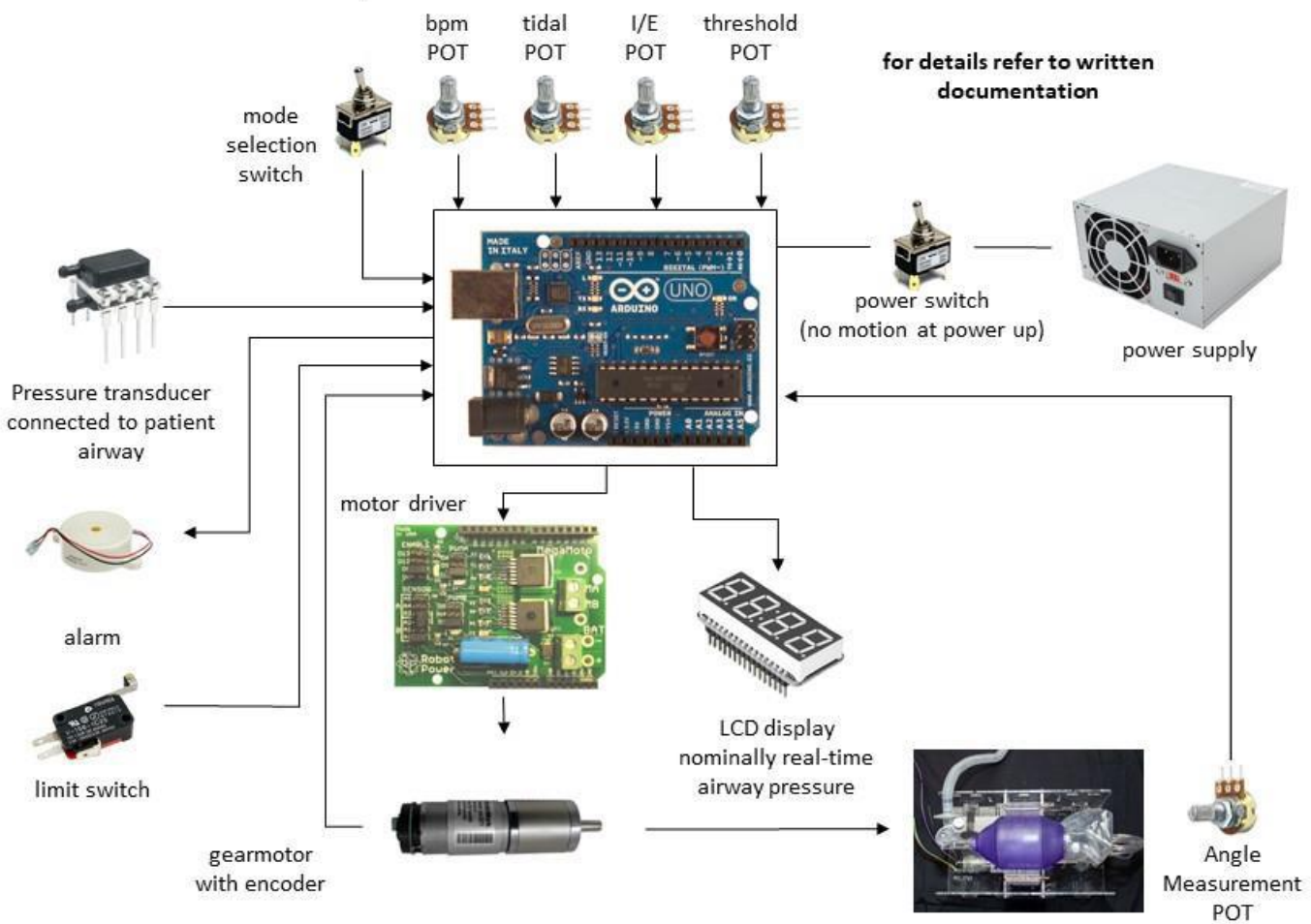

Figura 5. Arquitectura electrónica básica.

Figure 5. Basic electronic architecture.

En este esquema pude observarse los siguientes dispositivos:

1. Fuente de alimentación.

2. Placa de control de velocidad de motorreductor de Corriente Continua.

3. Motorreductor de Corriente Continua.

4. Microcontrolador. 
5. Fines de carrera.

6. Potenciómetro de medición de ángulo.

7. Medidor de presión.

8. Zumbador para identificación de alarmas.

9. Display.

10. Llaves y perillas para programación, carga de datos y puesta en marcha.

11. Luz ultravioleta y sensor.

El desarrollo electrónico utiliza dos microprocesadores que tienen duplicadas las funciones de medición y control. Uno actúa como maestro y el otro como esclavo. Ambos microprocesadores están interconectados por dos líneas digitales entre sí (llamadas, líneas de vida), de tal manera que ambos conozcan la información en tiempo real de lo que está ocurriendo en cada uno de ellos y puedan verificar que el microprocesador maestro está operando correctamente. En el caso de que por las líneas de vida se reconozca una pérdida funcional del maestro, el esclavo tomará el control generando a su vez una alarma de aviso en el sistema.

Asimismo, se coloca un tercer microprocesador que es el encargado de establecer el control de un display, las comunicaciones externas, las correspondientes alarmas lumínicas y auditivas y todo aquello que no sea control y medición de las variables 
respiratorias. Cada microprocesador de control y medición se comunica con este tercer microprocesador directamente.

El motorreductor no está redundado por razones de espacio por lo que es un elemento de altísima robustez de tal manera de garantizar la más alta confiabilidad. Esta manejado con un driver (puente $\mathrm{H}$ ) individual instalado en cada sub-plaqueta de microcontrolador. Este driver puede tolerar hasta 50V y $3 \mathrm{~A}$ de máxima, tolerando $6 \mathrm{~A}$ de pico transitoriamente.

El motorreductor se vincula a ambos microprocesadores a través de un relé doble inversor el cual es gobernado por el microprocesador esclavo. Originalmente este relé está en su posición normal cerrado, habilitando la conexión entre el motorreductor y el microprocesador maestro. Si el maestro pierde lectura, el esclavo detecta esto y toma el control el relé direccionándolo hacia sí mismo y continuando con la operación de tal forma de seguir el ciclo respiratorio que estaba programado.

La Figura 6 es un esquema en bloques que muestra la filosofía del diseño utilizada basada en la redundancia electrónica. 


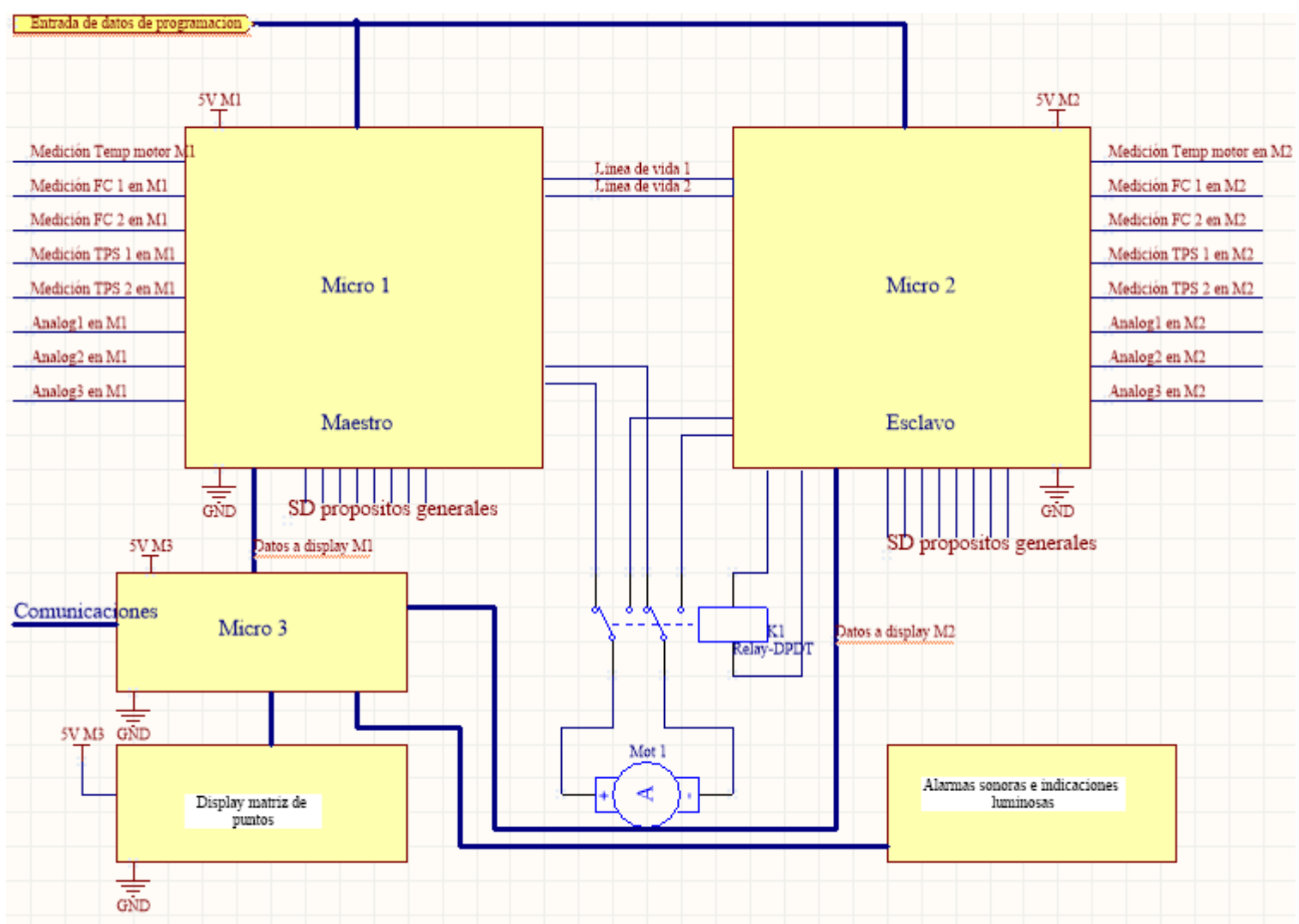

Figura 6. Esquema completo del circuito en bloques.

Figure 6. Complete circuit block diagram.

En la Figura 6 puede verse que cada microprocesador recibe los parámetros de programación simultáneamente. Para estar seguro de que cada uno tiene los mismos datos cargados es que se conectan las líneas de vida por las cuales se transfieren información, validando los procesos que están ocurriendo.

Una vez cargados y verificados los datos, una de estas líneas de vida funciona como un reloj de pulsos que le avisa al esclavo que el maestro está bajo control. Si estos pulsos se pierden, o si por alguna otra razón el AMBU no indica alguna de las 
variables sensadas, el esclavo toma el poder con el objetivo de intentar recuperar la secuencia de respiración.

Tal como se especificó anteriormente, cada microprocesador se comunica con un tercero, al cual le transfieren los datos para indicarlos en un display. Además, este tercer microprocesador recibe las alarmas originadas desde el maestro y/o el esclavo y las direcciona hacia un circuito de indicaciones luminosas y sonoras. Estas alarmas son mostradas también en el display.

Una vez ensamblado el conjunto entero, se coloca adentro de una caja metálica, con una tapa superior, la cual posee un amarre para ser transportada a mano (Figura 7).

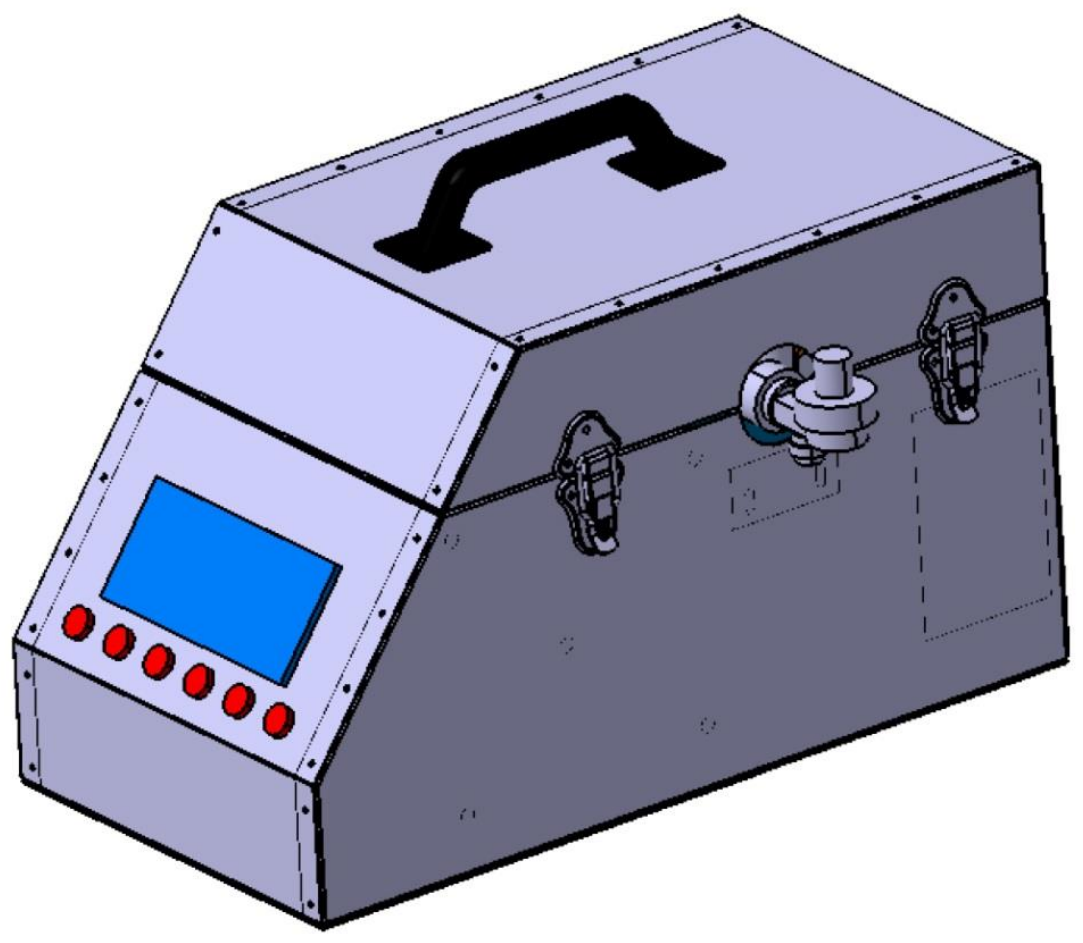

Figura 7. Respirador mecánico de emergencia.

Figure 7. Emergency ventilator. 
En su frente posee un panel frontal con un display digital para poder observar distintos parámetros y cuenta con la posibilidad de incorporar diferentes botones o potenciómetros para poder modificar los parámetros de la mecánica ventilatoria. Por su parte, los paneles laterales tienen ranuras de ventilación para facilitar la circulación de aire hacia su parte posterior y refrigerar así la electrónica y el motorreductor durante la operación. Adicionalmente, la caja posee aberturas para la conexión de los componentes del AMBU.

La caja contiene la placa electrónica y batería cerca de su parte frontal, debido a que tiene que estar conectada al display y a los elementos de comando, los cuales se encuentran en el panel frontal. Mientras que en la zona central, se ubican el mecanismo de accionamiento del AMBU junto con el motorreductor. Finalmente, en su parte posterior se encuentra la fuente de alimentación (Figura 8).

Al ser el AMBU un componente que sufre desgaste luego de los sucesivos ciclos de compresión, es necesario cambiarlo periódicamente. Por tal motivo, dicha operación debe ser sencilla y rápida. En consecuencia, el AMBU se encuentra próximo a la tapa de la caja, de forma tal que al quitarla, se accede inmediatamente al mismo. A su vez, los agarres del AMBU son lo suficientemente flexibles en su diseño para poder albergar distintos tipos AMBUs provenientes de diferentes fabricantes. 


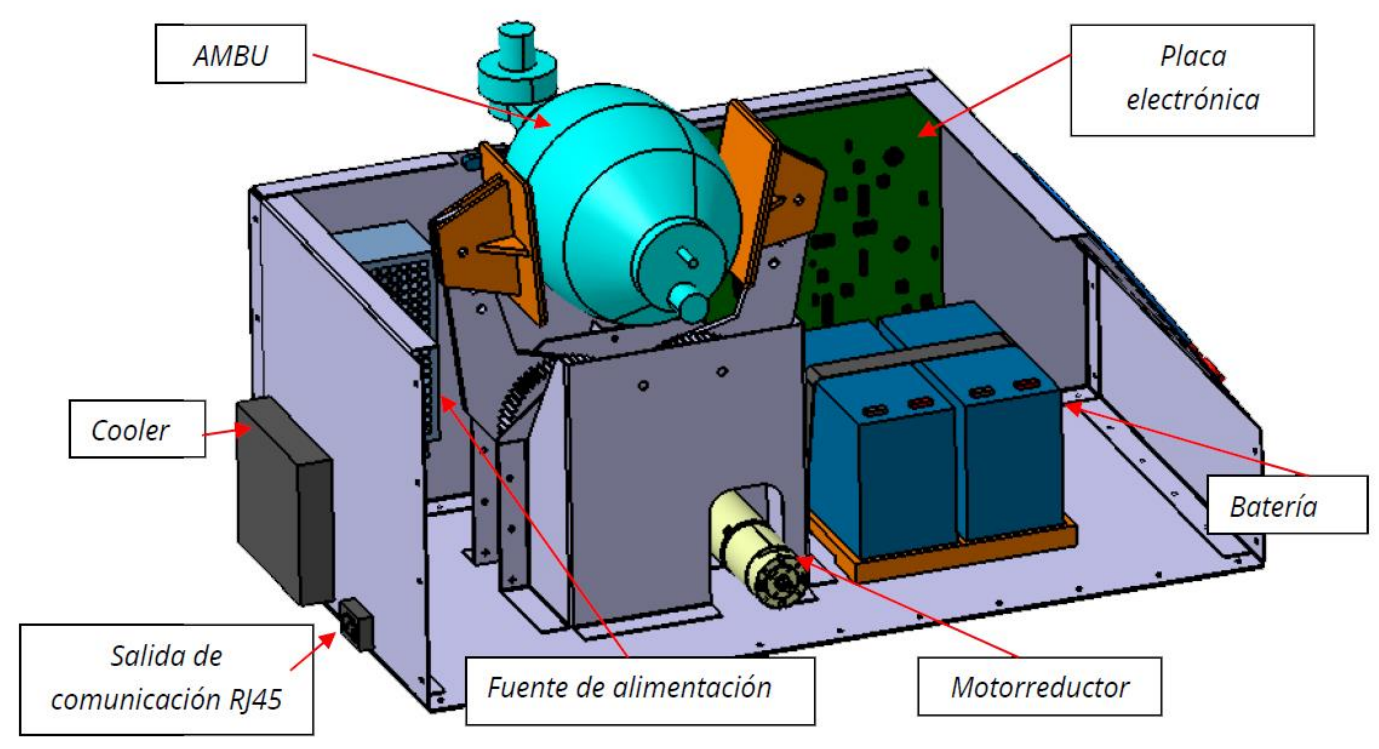

Figura 8. Interior del respirador mecánico de emergencia.

Figure 8. Inside of the emergency ventilator.

Durante el desarrollo del respirador, se efectuaron diferentes ensayos de durabilidad y funcionamiento. El primero de estos ensayos se realizó sobre una bolsa AMBU, a fin de establecer la repetibilidad de las curvas de presión generadas luego de sucesivas compresiones. Asimismo, mediante este ensayo se obtuvieron conclusiones respecto de la vida útil de la bolsa y del desgaste del material.

El ensayo se planteó estableciendo la peor condición de funcionamiento. Con lo cual, la frecuencia de accionamiento fue de 30 veces por minuto, es decir, la máxima establecida por los organismos de salud para pacientes con patología COVID-19. 
Durante dicho ensayo, la boquilla de la salida de aire del AMBU se encuentra regulada de tal forma que la apertura se produce únicamente cuando la presión en el interior del $A M B U$ es mayor a $40 \mathrm{~cm}$ de columna de $\mathrm{H}_{2} \mathrm{O}$. Este limitante se establece considerando que el límite de presión superior para estos pacientes es, precisamente $40 \mathrm{~cm}$ de columna de $\mathrm{H}_{2} \mathrm{O}$. Por lo tanto, la presión en el interior del AMBU durante el accionamiento, siempre se encuentra en su valor máximo. Finalmente, se estableció el recorrido de los brazos de accionamiento en el máximo posible, es decir, cuando los dos brazos alcanzan el centro del AMBU. Esta condición establece el máximo volumen de inspiración logrado para este conjunto de brazos con sus características geométricas particulares. Por lo tanto, el ensayo se realizó en las peores condiciones, máximo volumen y máxima presión.

En las Figuras 9 y 10 se presentan los dispositivos del ensayo. Se puede observar el AMBU y los dos brazos de accionamiento.

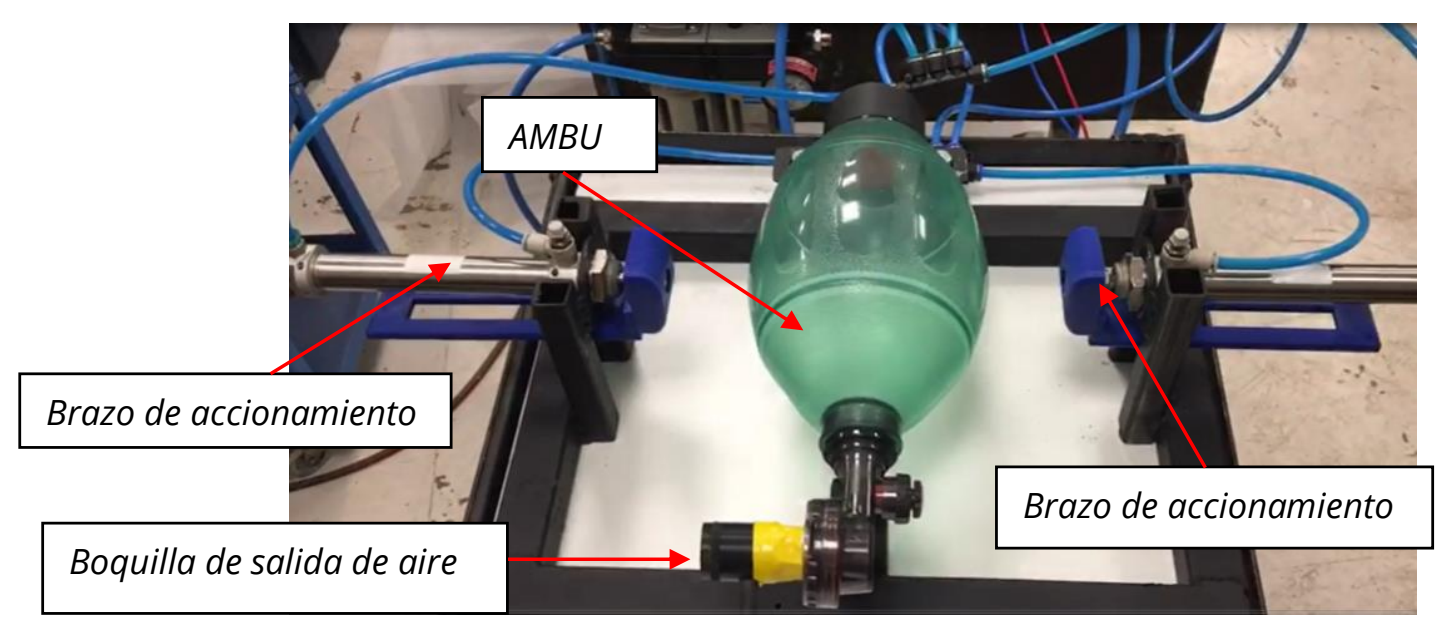

Figura 9. AMBU (relajado) en dispositivo de caracterización.

Figure 9. AMBU (unpressed) in characterization device. 


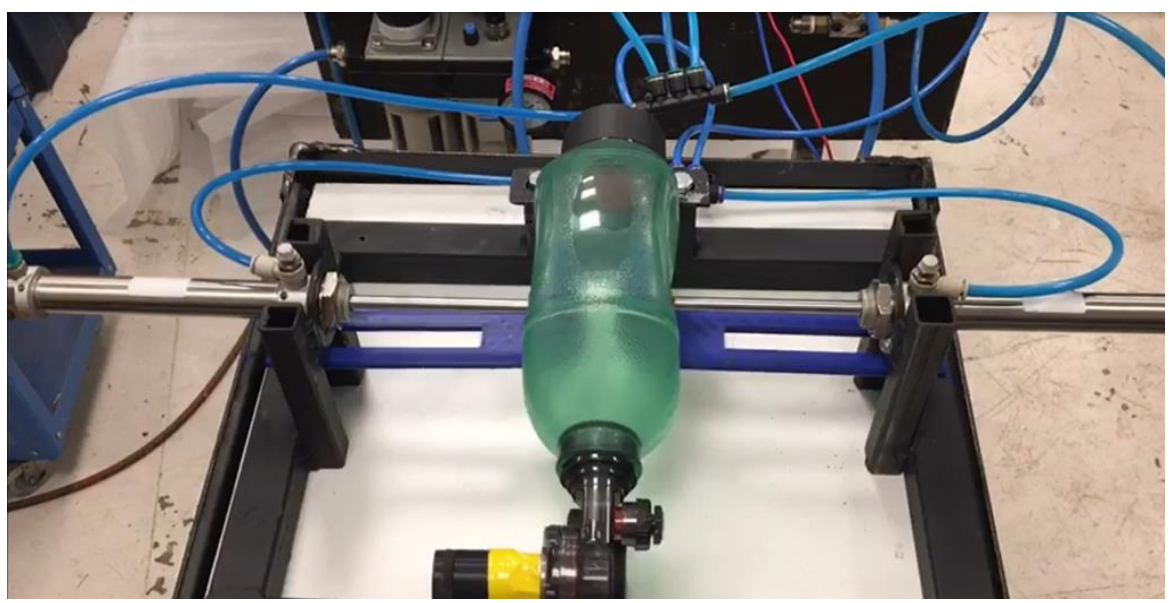

Figura 10. AMBU (apretado) en dispositivo de caracterización.

Figure 10. AMBU (pressed) on characterization device.

En la Figura 11 se presentan las curvas de presión obtenidas en la boquilla del AMBU registradas durante el ensayo. Se puede observar que la presión promedio durante el accionamiento del mismo se encuentra siempre cercana a los $4250 \mathrm{~Pa}(42,5 \mathrm{~cm}$ de columna de $\mathrm{H}_{2} \mathrm{O}$ )

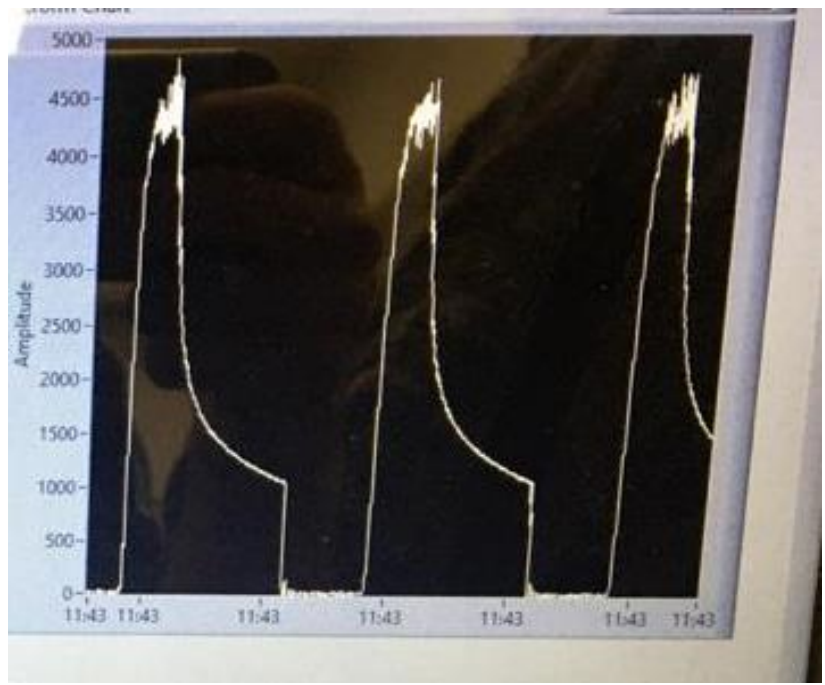

Figura 11. Curva de presión en un ciclo.

Figure 11. Pressure curve in one cycle. 
Innovación y Desarrollo Tecnológico y Social (2020) 2 (2): 134-166- Número especial COVID-19

En las Figuras 12 y 13 se indican las curvas de presión obtenidas en la boquilla del AMBU para el instante inicial y para diferentes cantidades de ciclos de accionamiento. Alcanzando un máximo de 310.000 ciclos.

Se observa que hay cambios en el funcionamiento del AMBU, lo cual se refleja en las curvas de presión registradas. Si bien las curvas tienen perfiles similares, el área bajo las curvas es cada vez menor, lo con significa que el volumen expulsado disminuye a medida que aumenta la cantidad de ciclos. No obstante el cambio en el volumen expulsado, se pude observar que los valores pico son similares para las diferentes cantidades de ciclos de accionamiento.

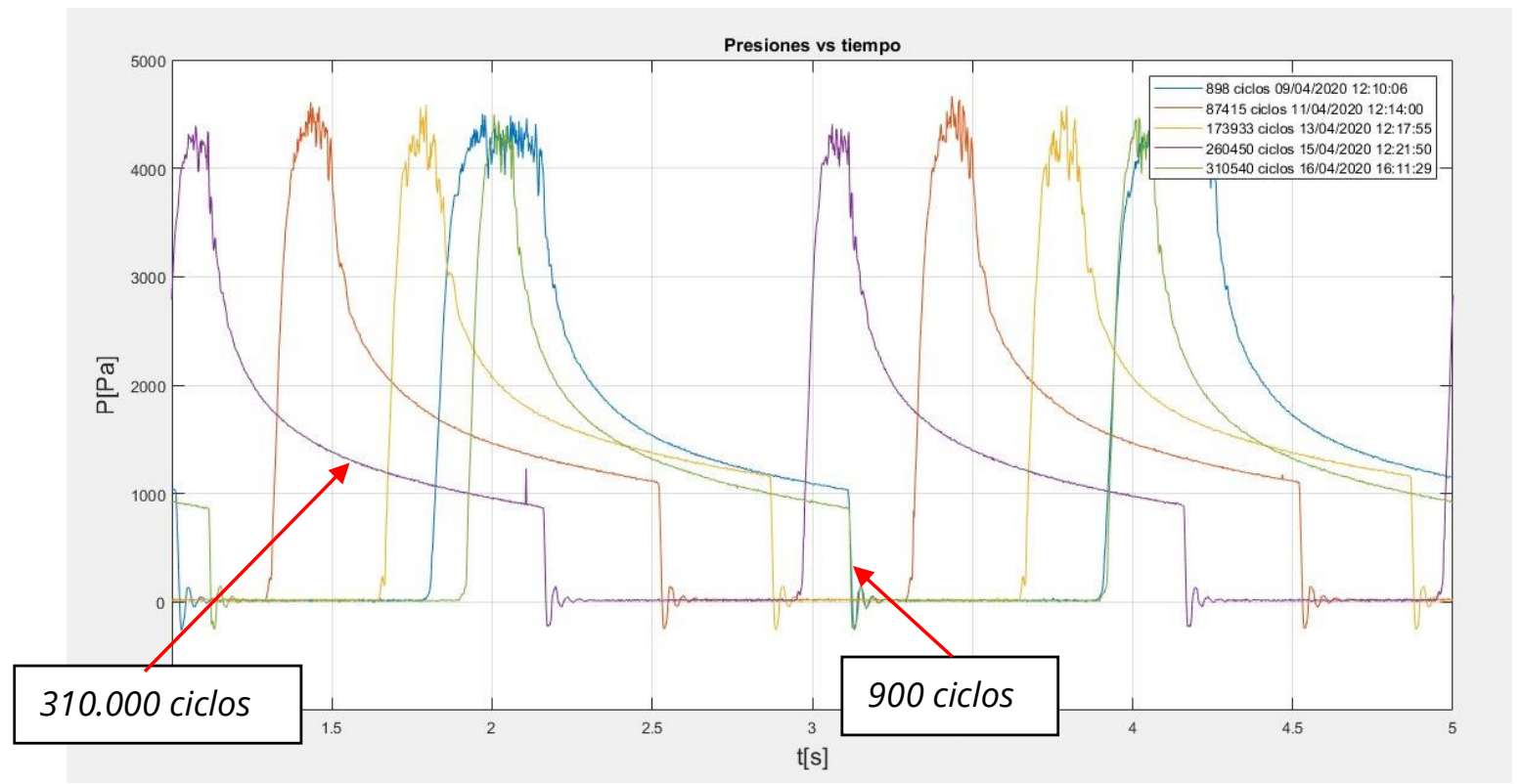

Figura 12. Curvas de presión en la boquilla de la bolsa AMBU para diferentes ciclos de accionamiento.

Figure 12. Pressure curves at the nozzle of the AMBU bag for different actuation cycles. 
En la Figura 13 se detallan las curvas de presión luego de aproximadamente 900 ciclos y luego de 310.000 ciclos. Se logra observar claramente la reducción en el área bajo la curva.

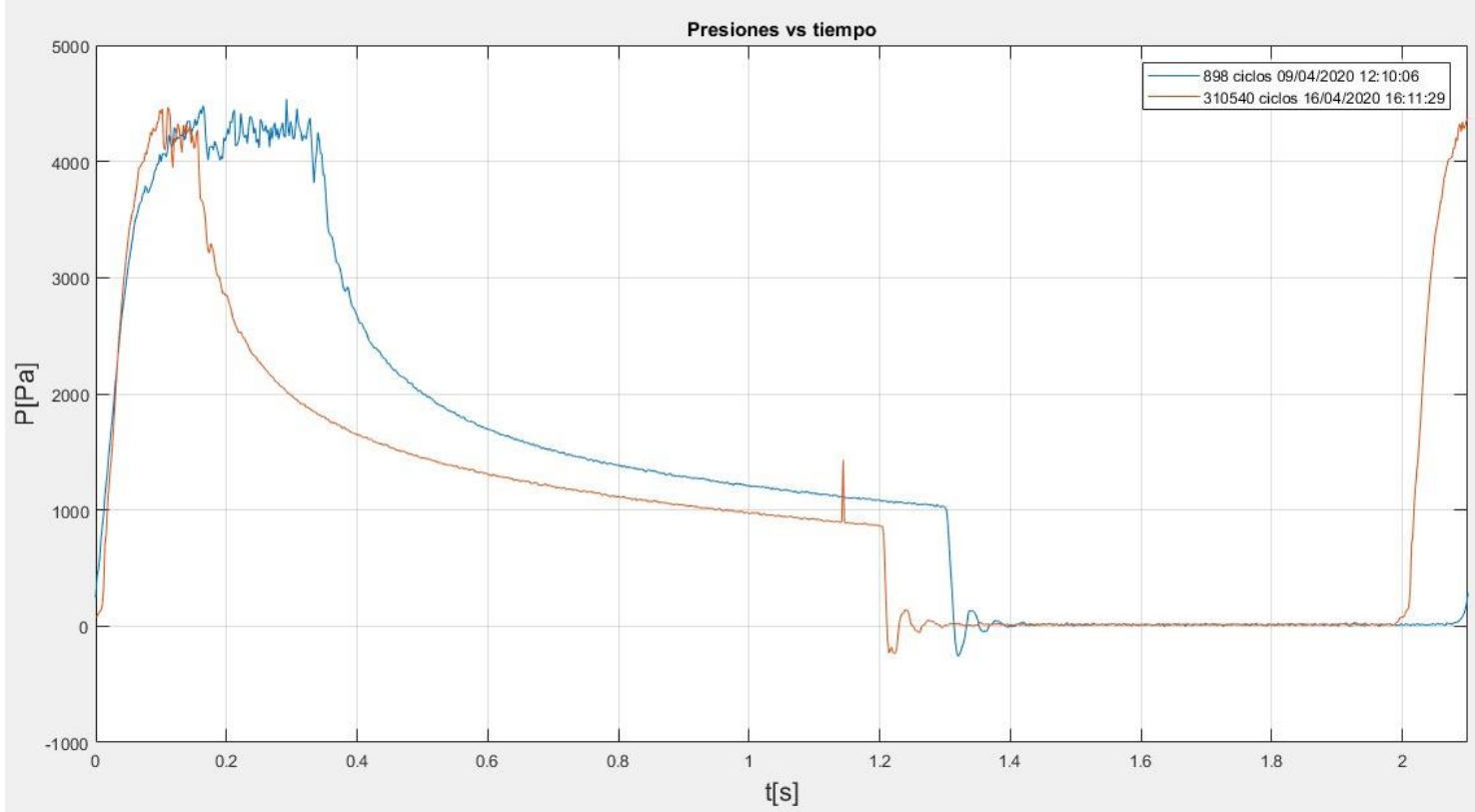

Figura 13. Detalle de curvas de presión en la boquilla de la bolsa AMBU.

Figure 13. Detail of pressure curves in the nozzle of the AMBU bag.

Luego de analizar los resultados, se concluyó que el cambio en las curvas de presión se debe principalmente a que luego de ciclar repetitivamente al AMBU, éste presenta una deformación remanente cada vez mayor, la cual no logra desaparecer en el corto tiempo que existe entre accionamientos. En la siguiente Figura 14 se observa el estado del AMBU y se aprecia la deformación mencionada. 
Innovación y Desarrollo Tecnológico y Social (2020) 2 (2): 134-166- Número especial COVID-19

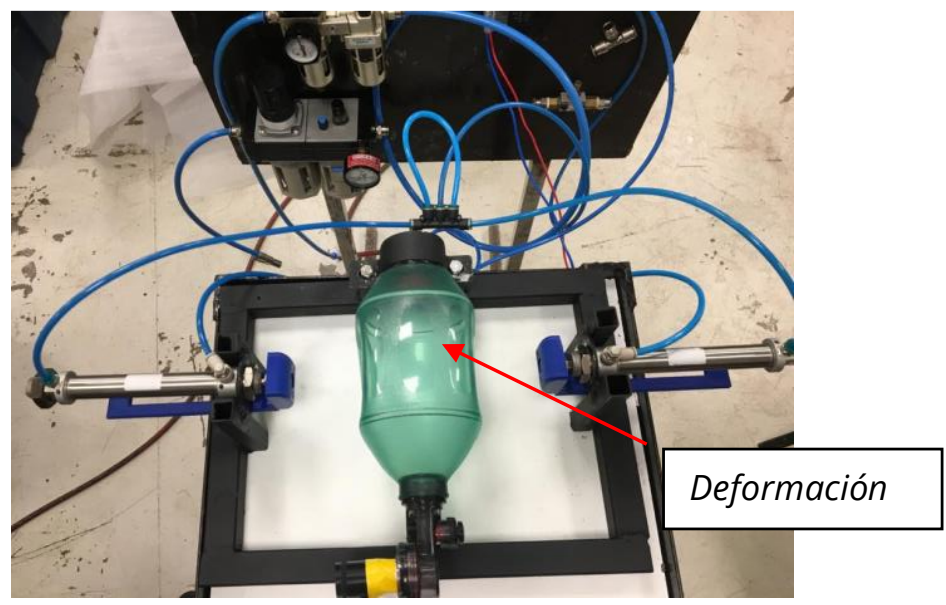

Figura 14. Deformación remanente del AMBU.

Figure 14. Remaining deformation of the AMBU.

Sin embargo, si se incrementa el lapso entre compresiones, permitiendo al AMBU recuperar su volumen inicial y luego se lo comprime nuevamente, se logra obtener curvas de presión similares a las iniciales, tal como se observa en la Figura 15.

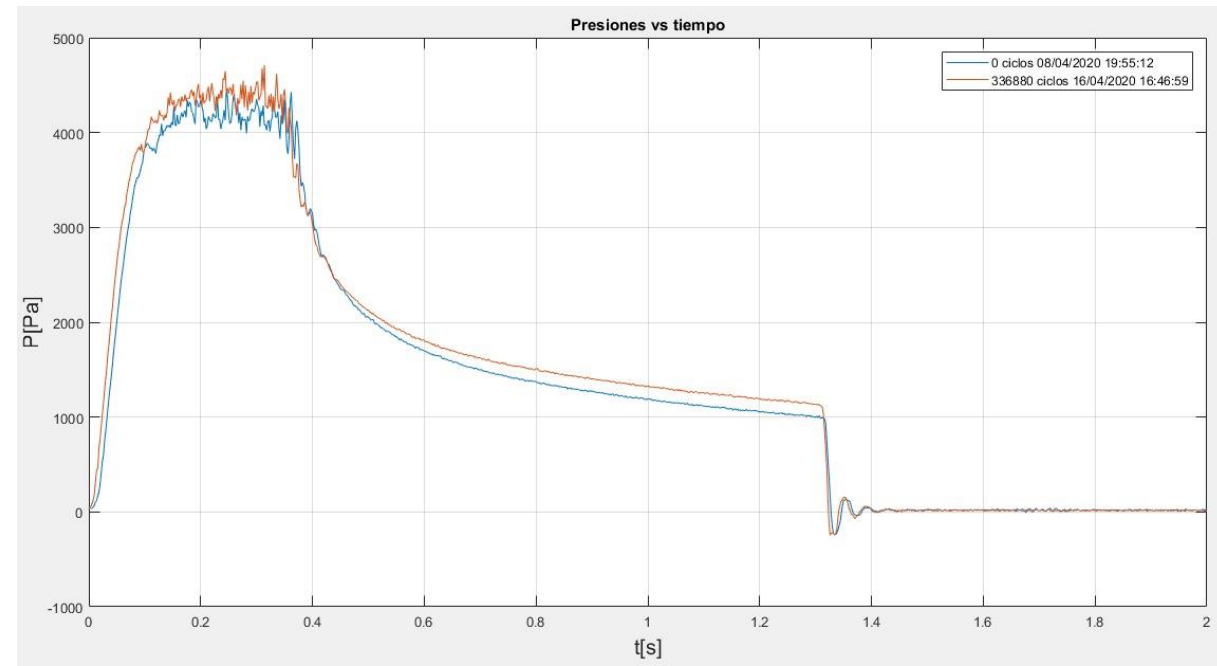

Figura 15. Comparación de curvas de presión permitiendo al AMBU recuperar su volumen inicial.

Figure 15. Comparison of pressure curves allowing the AMBU to recover its initial volume. 
Durante la etapa de ensayos del mecanismo de accionamiento, se observó que los engranajes de aluminio utilizados en el diseño del MIT Emergency Ventilator (MIT 2020) no soportaban el desgaste en los dientes de la corona dentada luego de sucesivos ciclos (Figura 16). Asimismo, este resultado se corroboró posteriormente con publicaciones que el mismo grupo de trabajo del MIT Emergency Ventilator (MIT 2020) público en su sitio web.

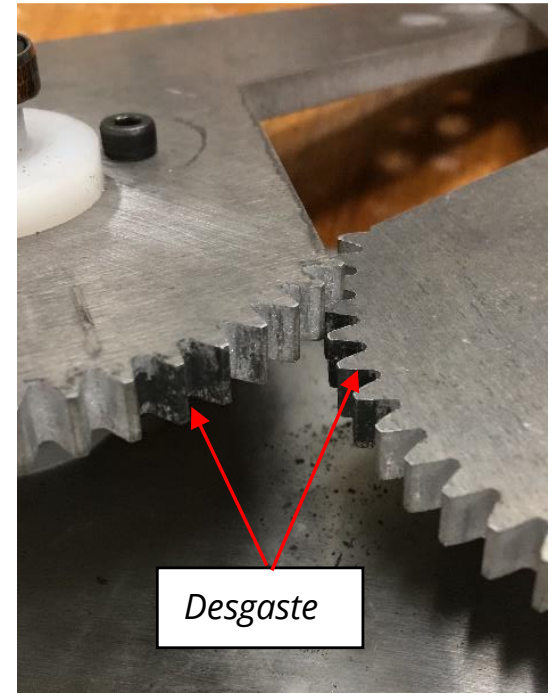

Figura 16. Desgaste en engranajes de aluminio.

Figure 16. Wear on aluminum gears.

En consecuencia, debido a la necesidad de utilizar otro material para los engranajes, se optó por realizar pruebas con engranajes de resina de acetal y de acero. Para ello, se realizaron los cálculos estructurales correspondientes para determinar el ancho del diente mínimo para cada uno de estos dos materiales.

La ventaja de usar engranajes de acero es su alta dureza y rigidez, mientras que su desventaja es el peso, las posibilidades de oxidación y la necesidad de lubricación 
cada cierto intervalo de tiempo. En cambio, los engranajes de acetal presentan menor propiedades mecánicas que los de acero, pero su gran ventaja es la posibilidad de lubricarlos únicamente al inicio de su funcionamiento sin la necesidad de lubricarlos nuevamente. Para establecer cuál de las dos opciones es la más indicada, se procedió a ensayar ambas propuestas.

Luego de más de 230.000 ciclos de accionamiento, los engranajes de acero no presentaron mayores inconvenientes más allá de un leve desgaste en uno de sus dientes. Se concluyó que este desgaste se debió a la escasa lubricación con la que se realizaron las pruebas.

En cuanto a los engranajes de resina de acetal, aún se encuentran en etapa de verificación de durabilidad, buscando que realicen la misma cantidad de ciclos de accionamiento que los engranajes de acero. Como una primera conclusión se puede observar que estos engranajes sufren mayor deformación elástica al momento del contacto entre sus dientes que los engranajes de acero. Esta deformación produce que aumente considerablemente la fricción entre ellos, incrementando el torque resistivo del sistema. La Figura 17 corresponde a un video, filmado en cámara lenta, donde se aprecia la deformación de los dientes de acetal al momento del engrane. Si bien el aumento del torque resistivo es un aspecto a considerar, los engranajes de acetal siguen siendo una opción viable siempre que el torque resistivo se encuentre entre los parámetros de diseño. También es importante resaltar que a medida que se producen los sucesivos engranes de dientes, gracias a que la resina 
acetal es un material blando, estos van sufriendo pequeños desgastes y acoplándose adecuadamente unos a otros, disminuyendo con el paso del tiempo el mencionado torque resistivo.

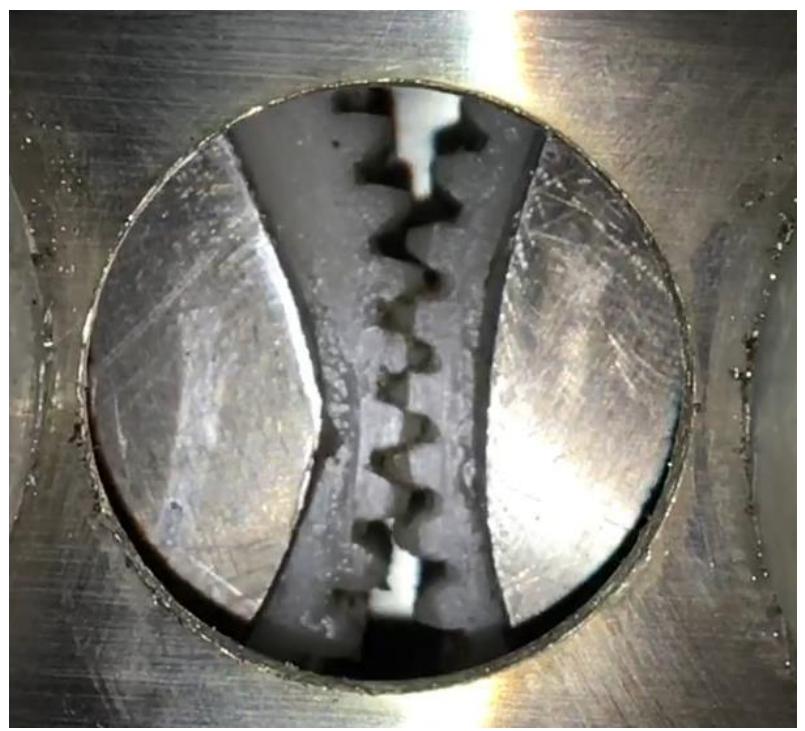

Figura 17. Engrane entre los dientes de resina acetal.

Figure 17. Acetal resin tooth contact.

Una vez alcanzado el número de ciclos estipulado, se observará si el desgaste se encuentra dentro de lo esperado y si no ocurrió ninguna falla, se optará por este material antes que el acero. Basando la elección principalmente en los aspectos de la lubricación y la oxidación antes mencionados.

Desde el punto de vista de los componentes electrónicos y sistemas de control, en una primera instancia se realizaron simulaciones de lectura de los parámetros a registrar por los sensores, de forma de corroborar su procesamiento y posterior 
Innovación y Desarrollo Tecnológico y Social (2020) 2 (2): 134-166- Número especial COVID-19

visualización en el display. En la Figura 18 se presentan las curvas de presión y volumen graficadas.

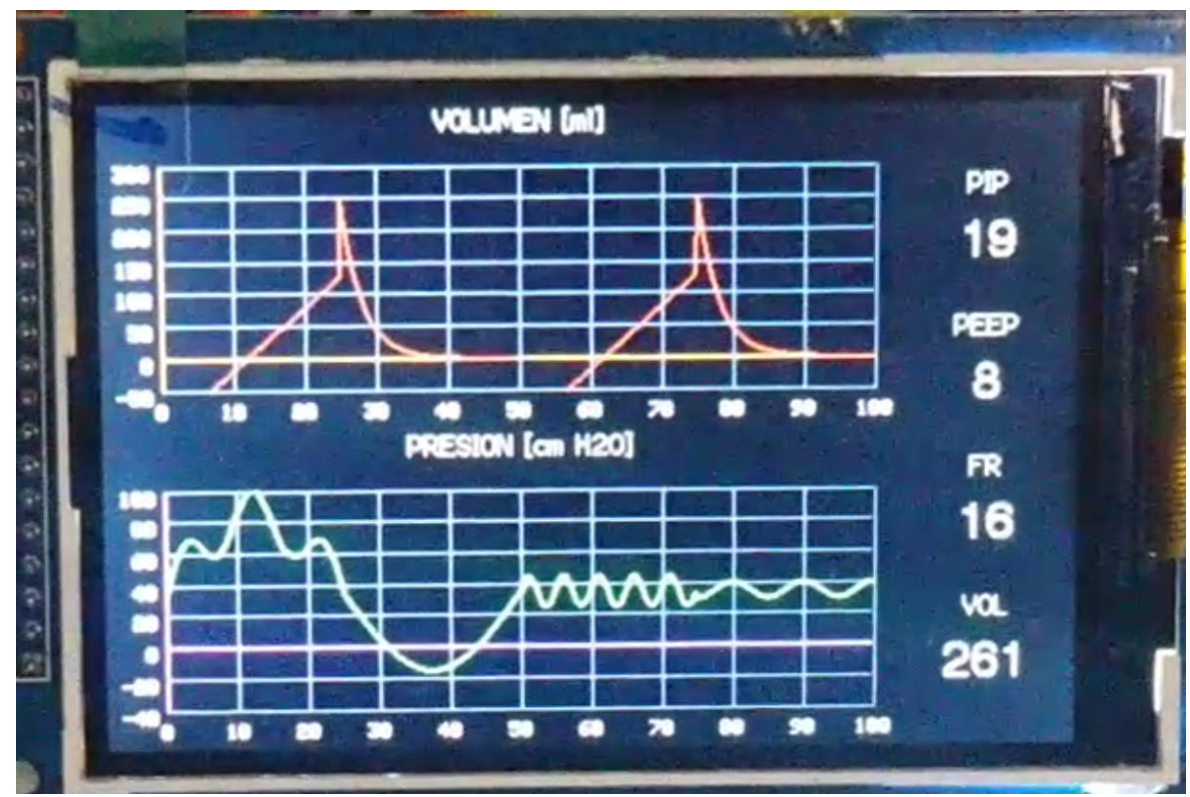

Figura 18. Curvas de volumen y presión graficadas.

Figure 18. Plotted volume and pressure curves.

En cuanto a los ensayos de funcionamiento del conjunto ensamblado, hasta el momento se realizaron pruebas sobre la lectura del grado de apertura de los brazos y sobre el control del motorreductor. En la siguiente figura se presentan las curvas de lectura del grado de apertura de los brazos, para 10, 20 y 40 grados, manteniendo en todos los casos una frecuencia de 30 respiraciones por minuto y una relación l:E 1:2. Se puede analizar que el control sobre el motorreductor logra el desplazamiento deseado de los brazos, reproduciendo fielmente el grado de apertura que es indicado por el operador. 
Innovación y Desarrollo Tecnológico y Social (2020) 2 (2): 134-166- Número especial COVID-19

Finalmente, en la Figura 19 se observa el mecanismo y el AMBU montado para el ensayo final de funcionamiento para obtener las últimas curvas presentadas.

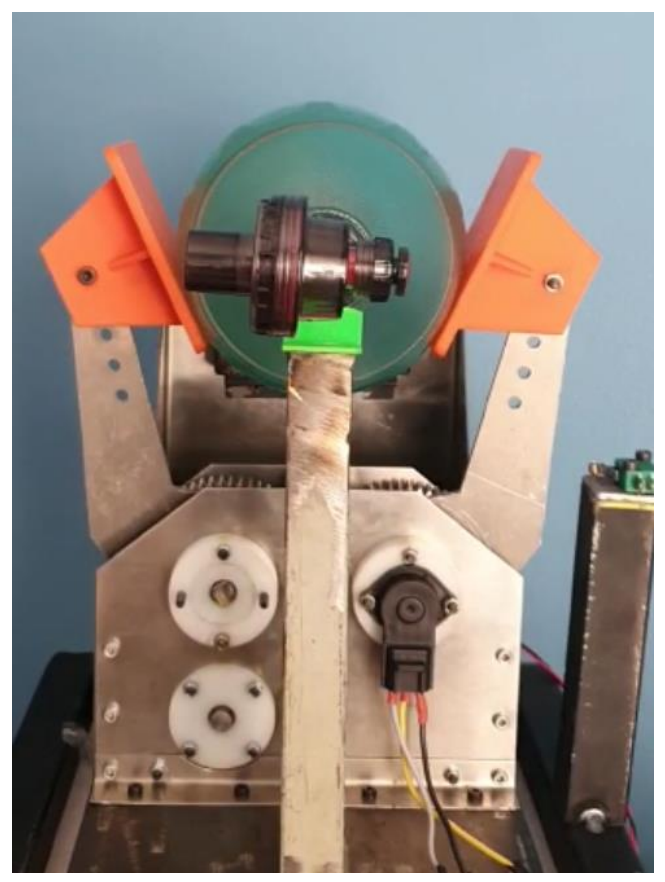

Figura 19. Ensayos sobre el mecanismo y el AMBU.

Figure 19. Tests on mechanism and AMBU.

Luego, ensamblando varios prototipos con todos sus componentes, se realizan pruebas para determinar el comportamiento del conjunto, logrando medir variables fluidodinámicas y electrónicas del respirador mecánico de emergencia. Permitiendo en consecuencia, conocer el funcionamiento del equipo en diferentes escenarios y determinar su vida útil.

Una vez superadas las etapas de ensayos, se espera que el respirador pueda cumplir de forma exitosa el objetivo planteado. 


\section{Financiamiento}

El desarrollo del respirador mecánico de emergencia posee el financiamiento del Centro Tecnológico Aeroespacial de la UNLP y la Agencia Nacional de Promoción de la Investigación, el Desarrollo Tecnológico y la Innovación, a través de la convocatoria IP COVID-19.

\section{Agradecimientos}

Agradecemos a los siguientes integrantes del grupo de trabajo que lleva adelante el proyecto del respirador mecánico de emergencia, compuesto por:

- Esp. Ing. Pablo L. Ringegni, Coordinador de la UIDET GEMA.

- Dr. Ing. Sebastián Delnero, Coordinador alterno de la UIDET LACLYFA, Director ejecutivo del Departamento de Aeronáutica.

- Ing. Luis Mariano Mundo.

- Ing. Diego Day.

- Ing. Elmar Mikkelson.

- Ing. Guillermo N. Garaventta.

- Ing. Daniel Hamann.

- Ing. Carlos F. Curell.

- Ing. Sergio Bustamante.

- Ing. Ariel Gamarra.

- Srta. Gabriela Tavera. 
Innovación y Desarrollo Tecnológico y Social (2020) 2 (2): 134-166- Número especial COVID-19

Asimismo, agradecemos a FC Servicio Metalúrgico Industrial y a todas las personas e instituciones que brindan su apoyo a este proyecto.

\section{Referencias bibliográficas}

ANPIDTYI (2020). Agencia Nacional de Promoción de la Investigación, el Desarrollo Tecnológico y la Innovación. Acciones sobre COVID-19. https://www.argentina.gob.ar/sites/default/files/covid-19 -ce - ip adjudicados.pdf Cairo, J.M. (2012). Pilbeam's Mechanical Ventilation: Physiological and Clinical Applications. Elsevier Mosby.

Hess, R.D. y Kacmarek, R.M. (2014). Essentials of Mechanical Ventilation. McGraw-Hill Education.

Medicines \& Healthcare Products Regulatory Agency (2020). Medical devices regulation and safety. Specification for ventilators to be used in UK hospitals during the coronavirus (COVID-19) outbreak.

https://www.gov.uk/government/publications/specification-for-ventilators-to-be-

used-in-uk-hospitals-during-the-coronavirus-covid-19-outbreak

MIT (2020). MIT Emergency Ventilator. Massachusetts Institute of Technology. https://emergency-vent.mit.edu/

Sociedad Argentina de Terapia Intensiva (2020). Mínimos clínicamente aceptables para el desarrollo de dispositivos capaces de dar soporte ventilatorio durante la pandemia generada por COVID-19. 


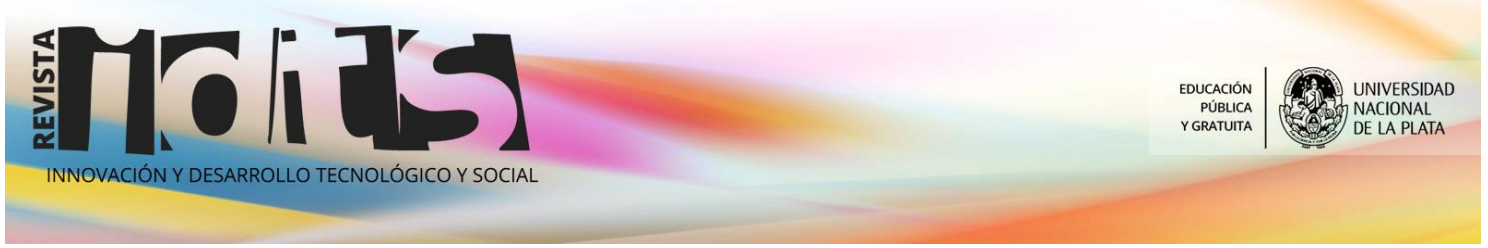

Innovación y Desarrollo Tecnológico y Social (2020) 2 (2): 134-166- Número especial COVID-19

https://www.sati.org.ar/images/SATI Minimos clinicamente aceptables para el de sarrollo de dispositivos capaces de dar soporte ventilatorio durante la Pandem ia generada por COVID 19.pdf

Tobin, M.J. (2006). Principles and Practice of Mechanical Ventilation. McGraw-Hill Medical Publishing Division. 